\section{An unusual case of immediate hypersensitivity reaction associated with an amalgam restoration}

\author{
B. Ilhan Kal, ${ }^{1}$ O. Evcin, ${ }^{2}$ N. Dundar, ${ }^{3}$ H. Tezel ${ }^{4}$ and I. Unal ${ }^{5}$
}
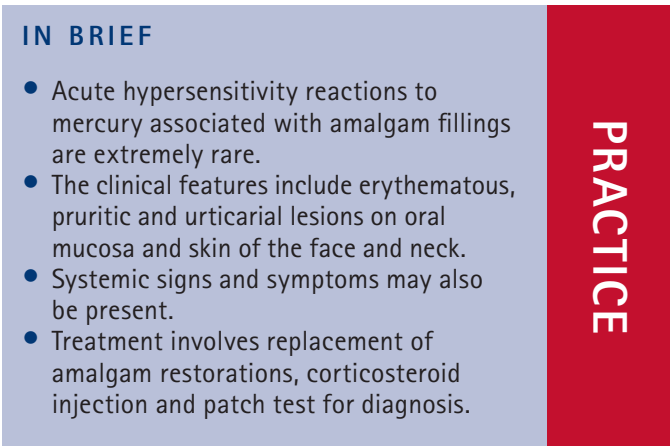

This report describes a case of immediate hypersensitivity reaction associated with the mercury component of amalgam restorations. The release of mercury induced an acute reaction which resulted in erythematous lesions, severe burning and itchy sensation and difficulty in breathing. Skin patch test results indicated a very strong positive reaction to mercury. Amalgam restorations were replaced with composite filling material. The patient, her family and her dental practitioner were strictly advised to use alternative restorative materials in case of a need for restoration.

\section{INTRODUCTION}

Hypersensitivity or allergy is a well recognised but rare adverse effect of materials that are routinely used in dentistry. These reactions are generally associated with dental restorative materials, anaesthetic agents, latex and antibiotics. ${ }^{1}$ Among restorative materials, amalgam is the most common dental material that induces hypersensitivity reactions of oral mucosa. ${ }^{1,2}$ The mercury component of the alloy has been mostly held responsible for adverse immunological effects, with occasional reactions to other components such as copper, tin or zinc. ${ }^{1,2}$

Hypersensitivity reactions to mercury associated with amalgam restorations comprise an array of clinical manifestations on oral mucosa. The most common type presents as a lichenoid reaction which involves a delayed, type IV and cell-mediated hypersensitivity

\footnotetext{
${ }^{1 *}$ Assistant Professor, ${ }^{3}$ Professor, Department of Oral Diagnosis and Radiology, Ege University School of Dentistry, 35100 Bornova-Izmir, Turkey; ${ }^{2}$ Assistant Professor, ${ }^{4}$ Professor, Department of Restorative Dentistry, Ege University School of Dentistry, 35100 Bornova-Izmir, Turkey; ${ }^{5}$ Professor, Department of Dermatology, Ege University School of Medicine, 35100 Bornova-Izmir, Turkey

${ }^{*}$ Correspondence to: Assistant Professor Betul Ilhan Kal Email: ilhanbetul@yahoo.com
}

\section{Refereed Paper}

Accepted 20 August 2008

DOI: $10.1038 /$ sj.bdj.2008.981

${ }^{\oplus}$ British Dental Journal 2008; 205: 547-550 response. ${ }^{1,3,4}$ The clinical and histopathological characteristics of lichenoid reactions are compatible with the disease lichen planus; however, the former present only in contact areas with dental restoration materials. ${ }^{3,5}$ Acute, more generalised or systemic reactions induced by type I hypersensitivity rarely occur and appear as red mucosal lesions with or without ulcerations in contact with the restoration or at other locations of oral mucosa. ${ }^{6,7}$ These reactions may be accompanied by systemic signs and symptoms, such as asthmatic attacks and urticaria of the skin. ${ }^{7}$ The delayed and immediate reactions to mercury associated with amalgam disappear or improve after removal of the restoration and do not recur if similar materials are avoided. ${ }^{1}$

This report describes a case of immediate hypersensitivity reaction associated with the mercury component of amalgam restorations in a female patient.

\section{CASE REPORT}

A 20-year-old girl was referred to the Department of Oral Diagnosis and Radiology after acute onset of extreme burning sensation and itchy lesions on the buccal mucosa and the skin on her neck, accompanied by difficulties in breathing.

Her mandibular right first and second molars had been restored with amalgam restorations on the same day at around 11am by her general dental practitioner. The occlusal restorations extended towards the buccal aspects of the molar teeth, which formed a contact surface between amalgam and the oral mucosa. No local anaesthetic agents were used during the procedure. An excessive burning and itchy sensation affecting the right buccal mucosa and the skin on the same side of the neck developed within two hours after dental treatment. The patient had difficulties in breathing and noticed a reddish area on her neck. She was immediately referred to our clinic by her practitioner and arrived around $2 \mathrm{pm}$. By the time she arrived the condition had become worse and she was extremely anxious, especially due to the difficulty in breathing. Her medical history was unremarkable; she was taking no medication and had not experienced any allergic reaction before. Extraoral examination revealed a large erythematous area covering the right side of the neck (Fig. 1). The patient had an intact and non-restored dentition except for the two recent amalgam restorations. An erythematous patch on the right buccal mucosa adjacent to the amalgam restorations and extending distally from the buccal commissura to the retromolar region was noticed (Fig. 2). The patient's oral hygiene was insufficient and periodontal examination revealed slight gingival inflammation. 


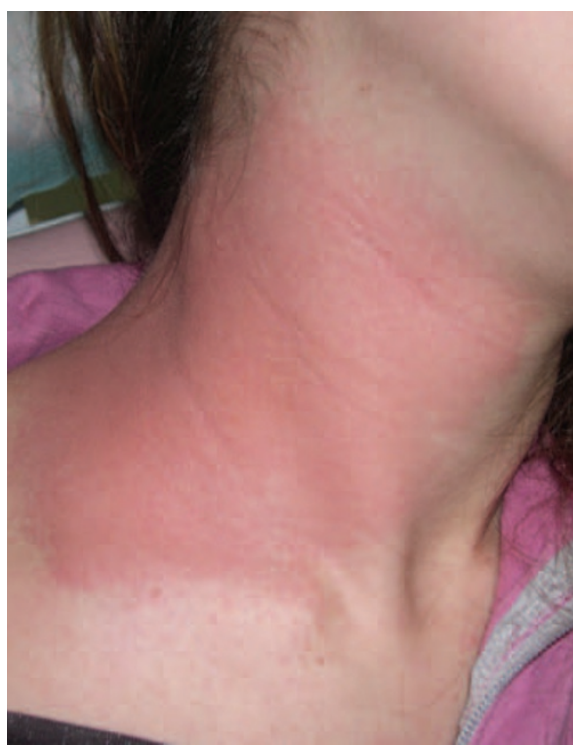

Fig. 1 Large erythematous area covering the right side of the neck

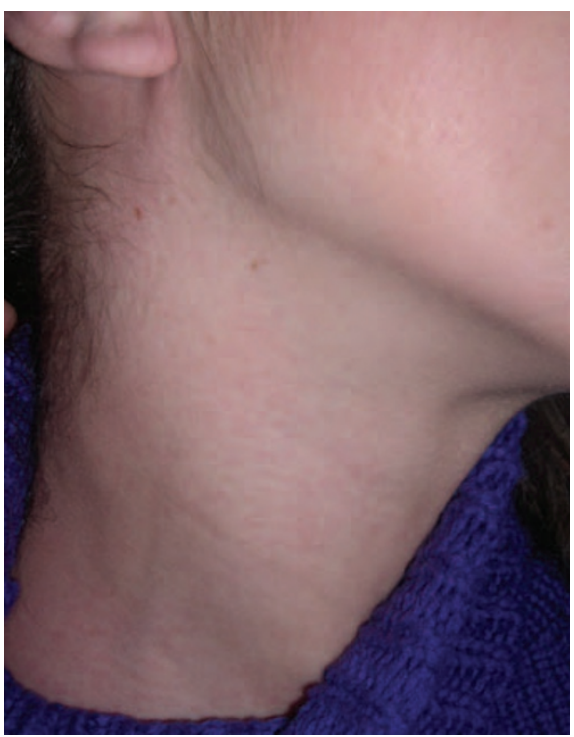

Fig. 3 The normal appearance of the skin covering the right side of the neck after removal of amalgam restorations

Since no local anaesthetic agents were used during her treatment, the initial diagnosis included an allergic reaction to one of the components of amalgam. She was immediately given cortisone (prednisolone, $40 \mathrm{mg} / \mathrm{IV}$ ) and both amalgam restorations were removed using rubber dam, water-spray and high speed suction in order to minimise exposure to any mercury that might be released. The teeth were restored with temporary filling materials. The patient appeared normal during the examination next day. The erythematous lesions had improved and she stated that the burning and itchy sensation began to disappear shortly after removal of the amalgam restorations (Fig.

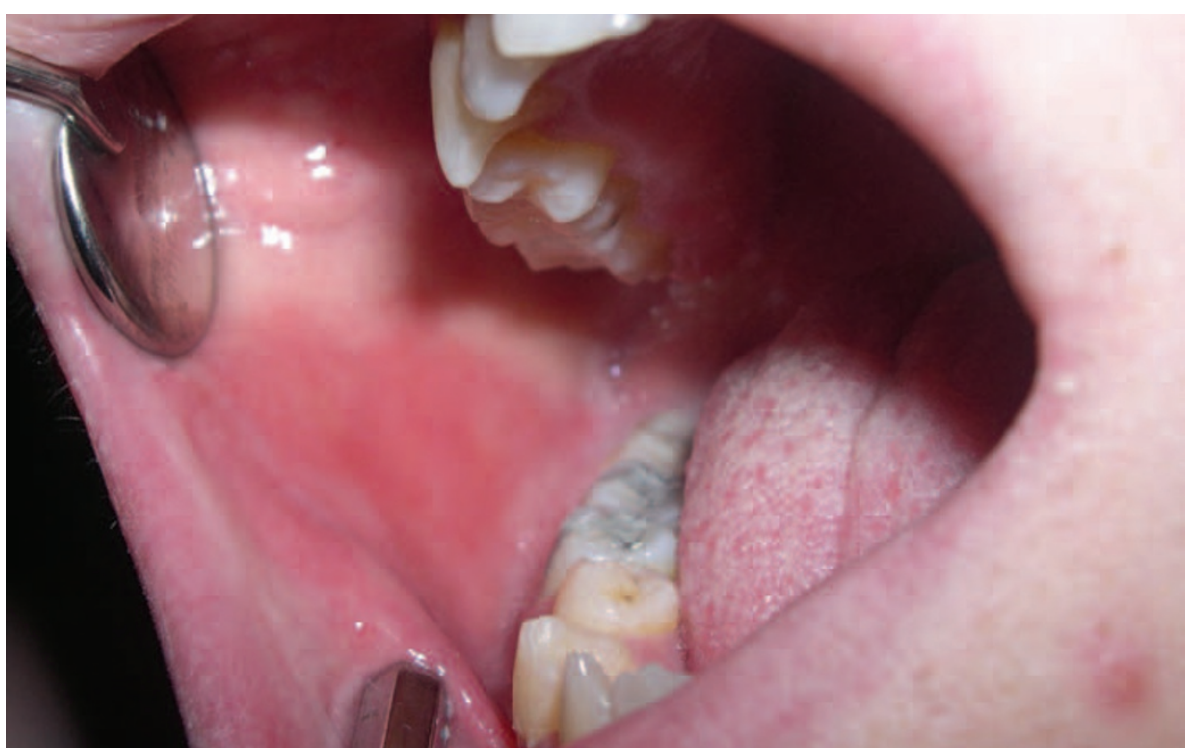

Fig. 2 The erythematous patch adjacent to the amalgam restorations extended distally from the buccal commissura to the retromolar region

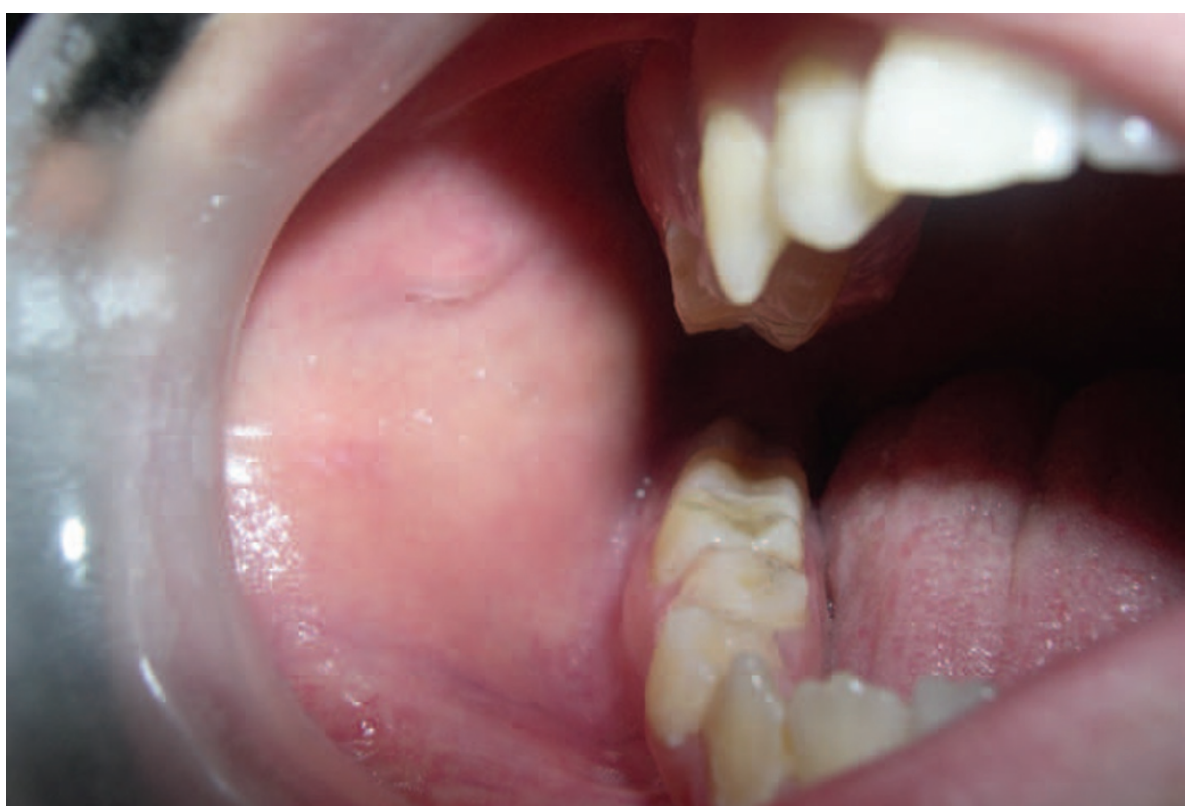

Fig. 4 The normal appearance of the oral mucosa on the next day and composite restorations

3). The mandibular right first and second molars were then restored with composite filling materials (Fig. 4) and the patient was referred for dermatological examination. Epicutaneous patch tests with 30 substances used in dental practice (Chemotechnique Diagnostics, Malmo, Sweden) and amalgam was performed to diagnose contact allergy. The allergens were applied within IQ Chambers and attached to the upper back. The patient was asked to wait in the clinic in order to perform an examination two hours after application of the allergens. She reported severe itching and burning sensations within 1.5 hours after the application and the tapes were removed by a dermatologist.
A very strong positive reaction to mercury with vesiculation and spreading erythamatous area was observed on the skin corresponding to the chamber containing mercury (Fig. 5). Eruption on an erythematous base was also observed on the area corresponding to amalgam (Fig. 6). The mercury and amalgam patches were removed and the rest of the allergens were re-applied. The patient was reviewed after 24 and 72 hours. No positive reactions to other allergens were observed. The patch test results indicated an acute hypersensitive reaction to mercury. The patient, her family and her dental practitioner was informed about the condition and they were strictly advised 
to use alternative restorative materials in case of a need for restoration in future.

\section{DISCUSSION}

The most common adverse immunologic effect of amalgam is related to contact allergy or delayed type IV hypersensitivity. ${ }^{1}$ The excessive immune response to an antigen leads to tissue damage and results in reddish, whitish or sometimes ulcerated lesions and lichenoid reaction pattern on the oral mucosa with an intimate anatomic relationship to the restoration..$^{1,3,7}$ Lichenoid lesions are the most prevalent amalgam-related delayed hypersensitivity reactions in the oral cavity. ${ }^{1,8}$ These reactions present similar clinical and histopathological characteristics to the disease lichen planus. They may appear as classical white lesions with Wickham striae, but often erythematous areas are observed., ${ }^{9,10}$ It has been suggested that lichen planus and lichenoid reactions represent a reaction pattern with T-cell-mediated immunologic mechanisms as a common trait. ${ }^{1,3}$ One of the two theories that have been proposed to explain the relationship between amalgam and the presence of lichenoid reactions was the galvanic reaction theory. It was suggested that the electrochemical current that flows between and within the restorations induced lichenoid changes on oral mucosa. ${ }^{10}$ However, the fact that the currents produced by the restorations in the oral cavity are too small to have any significant corrosive effect on the mucous membrane invalidated this theory. The second theory involves allergic contact hypersensitivity, which is mostly emphasised. ${ }^{3,10}$ However, although delayed type IV hypersensitivity reactions are often held responsible for the development of lichenoid changes, contact hypersensitivity to mercury and amalgam has not always been determined as contributing factors for the pathogenesis of oral lichenoid reactions. ${ }^{2}$ Contact hypersensitivity to mercury and amalgam, defined as a positive epicutaneous test to mercury, has been found in 8.3-78.9\% of the patients with amalgam-related oral lichenoid reactions. ${ }^{2,11}$ Studies investigating the effect of removing amalgam fillings in these patients have also produced variable results. A number of studies have indicated that the presence of a positive patch test result is a good predictor of lesion regression after amalgam replacement. ${ }^{3}$ On the other hand, some investigators reported improvement of the lesions after amalgam removal even though the patch test results were negative. ${ }^{5}$ Therefore, the pathogenesis of lichenoid lesions seems to be complex and is still not fully understood.

Immediate hypersensitivity reactions of the oral mucosa to amalgam restorations are far less common than delayed hypersensitivity reactions. ${ }^{6}$ These reactions tend to occur within hours of placement of a restoration and present as erythematous, pruritic, urticarial lesions typically on oral mucosa and the skin of the face and neck. Burning sensations associated with the formation of vesicles and systemic signs and symptoms, such as asthmatic attacks and urticaria of the skin may also be present., ${ }^{4,6}$ The clinical presentations of acute reactions to amalgam vary in reported cases. In the case reported by Ulukap1 ${ }^{12}$ an 8-year-old boy who presented with an erythamatous area on the buccal mucosa, cheek and neck two days after the application of an amalgam restoration was described. Since no systemic signs and symptoms were noted, the time interval between the restoration of the tooth and detection of the lesions was much longer than it was in our case. Recently McGivern et $a l .{ }^{6}$ reported a 57-year-old female patient who had episodes of intraoral blistering and a facial rash provoked by dental treatment. Their patient also had not experienced difficulties in breathing or other systemic symptoms. The condition was self-limiting and subsided in two to three days, due to a decrease in mercury release to a level not able to maintain the hypersensitivity reaction. ${ }^{13}$ It was suggested that the self-limiting nature of acute responses to restorative materials may eliminate the need for removal of the material, however the presence of difficulty in breathing and extreme burning and itchy sensations necessitated the removal of amalgam in our patient.

Similarly to the case reported by Ulukapı, ${ }^{12}$ erythamatous areas on the buccal mucosa and the skin of the neck was the only clinical finding in our patient. However, the development of vesiculation and a spreading erythamatous area 1.5 hours after application of mercury suggested a very strong positive reaction to mercury. Although the rapid onset of extreme burning and itchy sensations with erythamatous lesions and difficulty in breathing after insertion of amalgam restorations suggest type I hypersensitivity in our patient, the precise nature of these reactions is not clear. Type I hypersensitivity is a rapid allergic reaction that involves secretion of IgE by plasma cells which later binds to the receptor FceRI on the surface of tissue mast cells. ${ }^{6}$ After reexposure to the allergen, FceRI-associated IgE molecules bind allergen and aggregate, thereby activating mast cells. Activated mast cells secrete preformed mediators, including proteases and vasoactive amines such as histamine. ${ }^{14}$ The reaction may be either local or systemic. Localised reactions result in an urticarial rash and other changes, while severe reactions may be more widespread with oedema, tachycardia and respiratory difficulty. ${ }^{6}$ Type I hypersensitivity

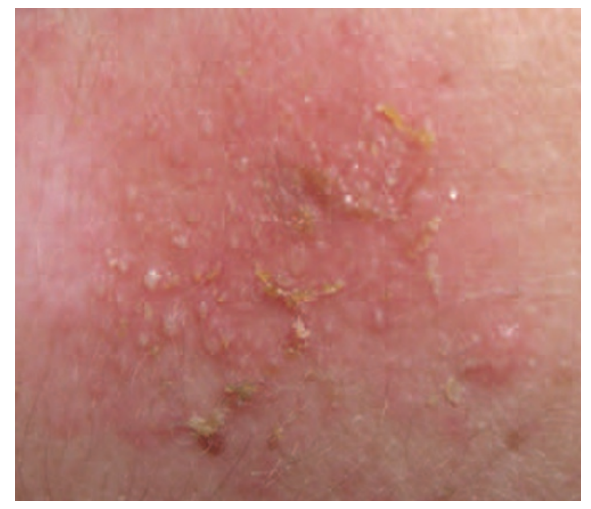

Fig. 5 Vesiculation and spreading erythamatous area 1.5 hours after application of a mercury chamber, indicating a very strong positive reaction to mercury

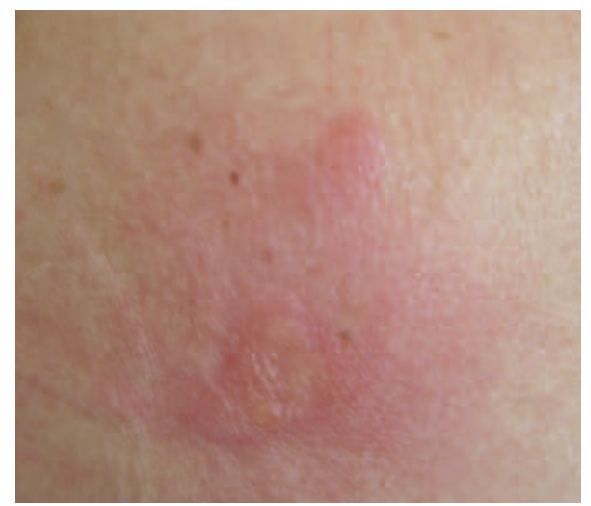

Fig. 6 Eruption on an erythematous base on the skin corresponding to amalgam 
responses are detected using the in vitro radioallergosorbent test (RAST) for antigen-specific IgE antibodies or by skin prick testing. ${ }^{6}$ The use of these assays is limited due to non-availability and toxicity of the mercury molecule, which makes it difficult to confirm that acute responses to mercury are the result of a type I hypersensitivity response. Nevertheless, as stated by McGivern et al., ${ }^{6}$ the clinical features of the reaction help to distinguish between acute and chronic responses to mercury.

Another notable point in our case is the difference between the responses to mercury and amalgam after the patch test. The response to amalgam was weaker than it was for mercury, which may lead to a discrepancy. However, it is stated that the reactions of the skin and oral mucosa are often different and although the reasons for it are not understood, it is possible for the skin to be sensitised but not the oral mucosa, there may be concurrent sensitisation of both skin and mucosa, or the mucosa may be sensitised but not the skin.,15 Therefore, the weaker response to amalgam compared to mercury remains as an unexpected and inexplicable finding.

Whether it is a delayed or an immediate hypersensitivity reaction, patients require prior exposure to the antigen to become sensitised. ${ }^{6,15}$ The most common sources of metallic mercury exposure include amalgam restorations and broken thermometers, while exposure to some inorganic and organic mercury compounds is possible through antibacterials, topical medications, topical antiseptics or disinfectant agents. ${ }^{16}$ It is not fully understood why some patients react and others do not, and furthermore whether the mercury released from amalgam restorations is able to sensitise a patient. ${ }^{7,15}$ It has been stated that most probably other sources of mercury contact are more common causes of sensitisation. ${ }^{4}$ Our patient had a non-restored dentition, meaning that she had not been exposed to mercury associated with amalgam until she had her mandibular molar teeth restored. Therefore she had most probably been sensitised through other sources of mercury such as mercury-containing wound disinfectants, antibacterials or topical medications.

In conclusion, an immediate hypersensitivity reaction to mercury associated with amalgam restorations is presented. The release of mercury from amalgam restorations induced an acute reaction and resulted in erythematous lesions, severe burning and itchy sensations and difficulty in breathing. Skin patch test was applied to diagnose contact allergy which revealed a very strong reaction to mercury. It must be emphasised that the reported case is extremely rare considering the widespread use of amalgam as a restorative material over the last century.
1. Axéll T. Hypersensitivity of the oral mucosa: clinics and pathology. Acta Odontol Scand 2001; 59: 315-319.

2. Khamaysi $Z$, Bergman R, Weltfriend S. Positive patch test reactions to allergens of the dental series and the relation to the clinical presentations. Contact Dermatitis 2006; 55: 216-218.

3. Thornhill M H, Pemberton M N, Simmons R K, Theaker E D. Amalgam-contact hypersensitivity lesions and oral lichen planus. Oral Surg Oral Med Oral Pathol Oral Radiol Endod 2003; 95: 291-299.

4. Duxbury A J, Ead R D, McMurrough S, Watts D C. Allergy to mercury in dental amalgam. Br Dent $J$ 1982; 152: 47-48.

5. Dunsche A, Kästel I, Terheyden H, Springer I, Christophers E, Brasch J. Oral lichenoid reactions associated with amalgam: improvement after amalgam removal. Br J Dermatol 2003; 148: 70-76.

6. McGivern B, Pemberton M, Theaker E D, Buchanan J A G, Thornhill M H. Delayed and immediate hypersensitivity reactions associated with the use of amalgam. Br Dent J 2000; 188: 73-76.

7. Holmstrup P. Oral mucosa and skin reactions related to amalgam. Adv Dent Res 1992; 6: 120-124.

8. Kato Y, Hayakawa R, Shiraki R, Ozeki K. A case of lichen planus caused by mercury allergy. Br J Dermatol 2003; 148: 1268-1269.

9. Wong L, Freeman S. Oral lichenoid lesions and mercury in amalgam fillings. Contact Dermatitis 2003; 48: 74-79.

10. Camisa C, Taylor J S, Bernat J R Jr, Helm T N. Contact hypersensitivity to mercury in amalgam restorations may mimic oral lichen planus. Cutis 1999; 63: 189-192.

12. Laine J, Kalimo K, Happonen R P. Contact allergy to dental restorative materials in patients with oral lichenoid lesions. Contact Dermatitis 1997; 36: 141-146.

13. Ulukapı I. Mercury hypersensitivity from amalgam: report of a case. ASDC J Dent Child 1995; 62: 363-364.

14. Frykholm K O. Allergy to mercury from amalgam restorations. Acta Odonto/ Scand 1957; 15: 61-70.

15. Galli S J, Nakae S, Tsai M. Mast cells in the development of adaptive immune responses. Nat Immuno/ 2005; 6: 135-142.

16. Dodes J E. The amalgam controversy: an evidence-based analysis. J Am Dent Assoc 2001; 132: 348-356.

17. Garner L A. Contact dermatitis to metals. Dermatol Ther 2004; 17: 321-327. 